\title{
Avaliação do padrão e das queixas relativas ao sono, cronotipo e adaptação ao fuso horário dos atletas brasileiros participantes da Paraolimpíada em Sidney - 2000
}

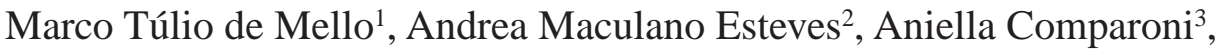 \\ Ana Amélia Benedito-Silva ${ }^{4}$ e Sergio Tufik ${ }^{5}$
}

\section{RESUMO}

Diversas metodologias têm sido desenvolvidas para investigar a qualidade e as principais queixas e distúrbios relacionados ao sono. Uma forma conhecida de investigar as características temporais dos organismos é a cronobiologia, ciência divide a população em três cronotipos básicos para avaliar as diferenças individuais na prevalência pelos horários de vigília e de sono: os matutinos, os vespertinos e os indiferentes. Outro ponto importante, é que existem poucos estudos relacionando o padrão do sono em indivíduos com necessidades especiais e a atividade física. O sono é considerado como restaurador e o exercício está associado diversas alterações no padrão de sono. A maioria dos estudos referente ao feito do exercício sobre o sono podem ser abordados ou correlacionados com a teoria de restauração das funções do sono. O objetivo deste estudo foi o de avaliar o padrão, queixas relativas ao sono, cronotipo e adaptação ao fuso horário de Sidney dos atletas brasileiros que disputaram a paraolimpíada em 2000. Participaram da avaliação 64 atletas paraolímpicos, com idades de 26,3 $( \pm 5,9)$. Todos os atletas responderam aos questio-

1. Professor Adjunto do Departamento de Psicobiologia/Unifesp-EPM.

2. Pós-Graduanda em Psicobiologia da Unifesp/EPM.

3. Estagiária Cepe/Unifesp, aluna do curso de Educação Física-Fefisa.

4. Pesquisadora e Professora da Associação Fundo de Incentivo à Psicofarmacologia (Afip).

5. Livre-Docente. Professor Titular do Departamento de Psicobiologia da Unifesp/EPM. Presidente da Afip.

Submetido em: 4/4/02

Versão revisada recebida em: 1/5/02

Aceito em: 19/5/02

Endereço para correspondência:

Prof. Dr. Marco Túlio de Mello

Departamento de Psicologia, Unifesp

Rua Marselhesa, 535

04020-060 - São Paulo, SP - Brazil

Tel.: (+55) (11) 5572-0177

E-mail: tmello@psicobio.epm.br nários de padrão e queixas relativas ao sono e cronotipo, passando também por uma adaptação ao fuso horário de Sidney. O processo de sincronização ao fuso horário foi realizado de forma abrupta, na tentativa de romper com o ciclo claro-escuro que estava relacionado ao horário brasileiro. Os resultados demonstram que $34,4 \%$ dos atletas apresentavam uma insatisfação com o seu próprio sono, sendo que os distúrbios de sono mais relatados foram: apnéia (14\%), refluxo gástrico $(15,6 \%)$, dor de cabeça $(14,1 \%)$, ansiedade pós-pesadelo $(39,1 \%)$, caimbras $(20,3 \%)$, sonilóquio $(26,6 \%)$, pânico noturno $(9,4 \%)$, PLM $(9,4 \%)$ e bruxismo $(9,4 \%)$. Em relação a avaliação do cronotipo dos atletas, $73,43 \%$ se demonstraram indiferentes, $6,22 \%$ vespertinos moderados e $20,31 \%$ matutinos moderados. Observou-se boa aceitação com todo o trabalho e o mesmo deve ter contribuído e refletido nos resultados finais dos jogos, visto que a equipe paraolímpica obteve um excelente resultado final.

Palavras-chave: Atletas paraolímpicos. Sono. Cronotipo. Exercício.

\section{ABSTRACT}

Evaluation of sleep patterns and sleep-related complaints, chronotype, and time zone adaptation of Brazilian athletes participant in the Sydney 2000 Paralympic Games

Several methods have been used to investigated the quality and the main complaints and disturbances related to sleep. Chronobiology is a way of investigating temporal features of organisms. Such science divides the population into three basic chronotypes to evaluate individual differences in the prevalence of wake and sleep times: the morning types, the evening types, and the indifferent. Another important aspect is the scarcity of studies that relate sleep pattern of the individuals to special needs and physical activity. Sleep is considered restorative, and exercise is associated to several changes in the sleep pattern. Most studies dwelling on the effects of exercise upon sleep may 
be correlated or seen under the light of the theory of restorative functions of sleep. The purpose of this study is to evaluate the pattern, the sleep-related complaints, the chronotype, and the time zone adaptation of Brazilian athletes who participated in the Sydney 2000 Paralympic Games. 64 athletes participated in the evaluation, mean age of 26.3 ( \pm 5.9). All of the athletes answered a questionnaire regarding sleep pattern, sleep-related complaints, chronotype, and time zone adaptation in Sydney. Time zone synchronization was abrupt, in an attempting at breaking the dark-light cycle related to the Brazilian time zone. Results show that $34.4 \%$ of the athletes were dissatisfied with their own sleep, and sleep disturbances most reported were: apnea (14\%), gastric reflux (15.6\%), headache (14.1\%), pre-nightmare anxiety (39.1\%), cramps (20.3\%), sleep-talking $(26.6 \%)$, nocturnal panic $(9.4 \%)$, periodic legs movement (9.4\%), and bruxism (9.4\%). As regards athlete chronotype, $73.43 \%$ showed to be indifferent, $6.22 \%$ were moderate evening type, and $20.31 \%$ were moderate morning type. Good acceptance of the work was observed, which may have contributed towards, and reflected in, the final results of the games, as the Brazilian team had an excellent final result.

Key words: Paralympic athletes. Sleep. Exercise. Chronotype.

\section{INTRODUÇÃO}

\section{Sono}

Os estudos sobre sono iniciaram-se no século XIX, com o desenvolvimento de aparelhos eletrofisiológicos necessários para estudar os potenciais de pequena amplitude.

Um grande passo nos estudos envolvendo o ciclo vigília-sono foi dado a partir da possibilidade de se estabelecer uma correlação entre os padrão comportamental e o eletroencefalográfico. O traçado típico da vigília caracteriza-se por ondas de baixa amplitude e de alta freqüência, enquanto que, à medida que se aprofunda, o sono apresenta ondas de voltagens crescentes e freqüências decrescentes, caracterizando fases distintas e que se ciclam durante o período em que o indivíduo dorme ${ }^{1}$.

Até ao momento o sistema de classificação dos estágios do sono mais amplamente aceito é o de Rechtschaffen e Kales ${ }^{1}$. Uma premissa importante desse sistema de classificação é de que o sono REM (rapid eyes movement), o SOL (sono de ondas lentas) e a vigília são fenômenos fundamentalmente diferentes, determinados por variáveis eletrográficas e fisiológicas, e de que o REM e o SOL se alternam durante a noite. Nem o sono nem a vigília são considerados processos unitários, sendo possível subdivisões praticamente ilimitadas para qualquer estágio.
De acordo com o sistema internacional, que vigora desde a década de $90^{2}$, os distúrbios de sono são classificados em:

- Dissonias: distúrbios intrínsecos do sono; distúrbios extrínsecos do sono; distúrbios do sono relacionados ao ritmo circadiano;

- Parassonias: distúrbios de despertar; distúrbios de transição sono/vigília; outras parassonias, como bruxismo noturno;

- Distúrbios do sono associados a doenças médicas e psiquiátricas;

- Novas propostas de distúrbios do sono.

\section{Cronobiologia}

A cronobiologia, ciência que investiga as características temporais dos organismos vivos ${ }^{3}$, inclui o estudo dos ritmos biológicos caracterizados pela recorrência, a intervalos regulares, de eventos bioquímicos, fisiológicos e comportamentais ${ }^{4}$.

Os ritmos biológicos persistem mesmo na ausência de pistas temporais, os chamados zeitgebers ${ }^{4}$. Essa persistência da ritmicidade biológica, em ambientes naturais ou artificiais mantidos constantes, é uma das demonstrações do caráter endógeno dos ritmos biológicos, isto é, do fato de serem determinados por fatores internos ao organismo.

Quanto aos ritmos não-circadianos, eles são agrupados em infradianos, que são ritmos de baixa freqüência com períodos maiores que 28 horas, como o ciclo menstrual, o ritmo de produção de plaquetas no sangue, cujo período é da ordem de sete dias, ou, no extremo, o ciclo reprodutivo do bambu chinês, cujo período é da ordem de 100 anos e em ultradianos, que são oscilações de alta frequiência com períodos menores que 20 horas, incluindo-se períodos da ordem de milissegundos, como o ritmo de disparo de neurônios ou da ordem de minutos, como o ritmo de batimentos cardíacos 5 .

O ritmo da temperatura central é considerado um sinalizador para os ritmos circadianos. A temperatura do corpo decresce para um valor mínimo durante o sono em torno das quatro horas da manhã e começa a crescer antes do início da vigília. A amplitude é da ordem de $0,4^{\circ} \mathrm{C}$ a $0,5^{\circ} \mathrm{C}$ em adultos jovens. O ritmo da temperatura é influenciado principalmente pelo sono e pelo exercício. Existe ainda uma correlação inversa proporcional entre temperatura corporal e liberação de melatonina.

Segundo Horne e Ostberg 6 , autores de um questionário para avaliar diferenças individuais na preferência pelos horários de vigília e de sono, a população humana pode ser dividida em três cronotipos básicos: os matutinos, os vespertinos e os indiferentes. Os matutinos, que podem ser matutinos extremos ou moderados, constituem 10 a $12 \%$ da população. Os indiferentes não têm horários preferen- 
ciais para dormir ou acordar. As fases dos seus ritmos endógenos são intermediárias, relativamente às dos matutinos e dos vespertinos.

Além da matutinidade e vespertinidade, deve-se levar em conta, em estudos cronobiológicos, as diferenças individuais quanto à duração do sono. Existem os pequenos dormidores - indivíduos que precisam de, no máximo, 6 h30 de sono - e os grandes dormidores - os que necessitam de, no mínimo, 8h30 de sono. Os dois tipos (pequenos e grandes dormidores) podem combinar-se de diversas maneiras com os cronotipos: há pequenos dormidores matutinos, pequenos dormidores vespertinos, grandes dormidores indiferentes ${ }^{6}$.

\section{Sono e pessoas portadoras de necessidades especiais}

De Mello et al. ${ }^{7}$ avaliaram o padrão e as queixas de sono em indivíduos portadores de deficiência por secção total da medula através da aplicação de um questionário. Foram avaliados 59 portadores de secção medular divididos em desportistas e não desportistas e os resultados da pesquisa constataram que a categoria de desportistas tiveram uma maior variabilidade de média de horas dormidas ao longo da semana. Foram encontradas diferenças significativas entre desportistas e não desportistas na incidência de ronco $(20 \%$ e $47 \%)$, despertares noturnos $(64 \%$ e $35 \%)$ e movimentos de membros inferiores (72\% e $38 \%)$. Estes resultados podem ser explicados por uma maior excitabilidade dos circuitos intrínsecos da medula, gerando tônus muscular e automatismos maiores na categoria de desportistas.

Outros estudos realizados por De Mello ${ }^{8}, \mathrm{Lee}^{9}$ e Yokota $^{10}$ enfocam especificamente a incidência dos Movimentos Periódicos de Pernas (PLM) em paraplégicos.

Pesquisas relacionando sono e deficiência mental vem sendo alvo de investigação durante os últimos 15 anos e tem-se demonstrado que estes possuem sérios problemas de sono. Estes problemas podem causar fardos emocionais e físicos durante a vigília, interferindo assim nos processos de aprendizagem e desempenho profissional. Foram realizados vários estudos para avaliar o comportamento destes indivíduos e seus distúrbios do sono ${ }^{11}$.

Outros estudos mostram uma diminuição significante de sono REM e um predomínio de sono não REM indiscriminadamente nos deficientes mentais ${ }^{12}$.

Estudos relacionando o padrão de sono dos deficientes com a atividade física são pouco abordados na literatura. Isso talvez se deva ao fato de que os padrões de sono dos deficientes não se diferencie em relação ao sono de indivíduos não portadores de necessidades especiais. Queixas do padrão de sono ocorrem nas duas populações. No entanto, quando observamos alguns aspectos específicos, podemos observar uma grande alteração na forma de ex- pressão do sono REM ou Paradoxal em indivíduos com deficiência visual. Os indivíduos cegos congênitos apresentam algumas diferenças durante o sonho, pois não apresentam movimentos oculares durante o sonho e sim alterações olfativas, táteis e auditivas durante o sonho. Em indivíduos portadores de deficiência visual adquirida, essa condição se reduz e chega a ser suprimida conforme o tempo e a idade em que se instalou a deficiência.

O sono é considerado como restaurador e o exercício está associado à alterações no padrão do sono. Existem várias facetas sobre a influência do exercício em relação ao sono. Estes incluem a intensidade e duração do exercício, o intervalo entre o fim da realização do exercício e o início do sono. Alguns fatores podem alterar o sono após a prática de atividade física, como a idade, aptidão física e massa corporal magra. A maioria dos estudos do efeito do exercício sobre o sono podem ser interpretados com papel importante na teoria de restauração das funções do sono ${ }^{13}$.

\section{Sono e exercício}

Os efeitos do exercício físico nos padrões de sono já vem sendo estudados a aproximadamente 30 anos. Em 1970 Heinzelmann e Bagley ${ }^{14}$ estudaram a capacidade de um programa de atividade física na promoção de comportamentos saudáveis. O treinamento teve duração de 18 meses, dividida em três sessões semanais de uma hora; os participantes relataram menor necessidade de sono, assim como um sono mais relaxado e restaurador.

Vuori et al. ${ }^{15}$ realizaram um levantamento epidemiológico em que foram entrevistadas 1600 pessoas, com idade variando entre 31 e 50 anos, com o objetivo de diagnosticar a influência da atividade física no sono. Os autores relatam que fatores sociais, psicológicos, condições do local em que dormem, o padrão de sono, o estilo de vida e as condições de vida do indivíduo influenciam diretamente na qualidade de sono e no desempenho físico. No entanto, o exercício moderado e vigoroso traz aspectos benéficos para a qualidade de sono.

Trinder, Montgomery, Paxton ${ }^{16}$, observaram as alterações e o tempo total de cada estágio do sono, bem como a eficiência do sono após a prática de exercício físico agudo ou crônico. Os autores relatam que durante o processo inicial de atividade física, os estágios 3 e 4 apresentam um percentual maior do que na fase basal; porém, com o processo de adaptação ao exercício físico, o organismo se reestrutura e volta a ciclar os estágios de sono normalmente.

Youngstedt ${ }^{17}$ analisou a curva resposta do ritmo circadiano comparando o exercício físico e a luz intensa, e seus resultados sugeriram que o exercício tem uma influência significativa no sistema do ritmo circadiano semelhante aos efeitos da luz. 


\section{OBJETIVO}

Avaliar o padrão, queixas relativas ao sono, cronotipo e adaptar ao fuso horário de Sidney os atletas brasileiros que disputaram a paraolimpíada em 2000.

\section{METODOLOGIA}

Foram avaliados através da aplicação de questionários 64 atletas paraolímpicos, com idades de 26,3 $\pm 5,9$, que disputaram as seguintes modalidades desportivas: natação, basquete, futebol PC, halterofilismo, ciclismo, tênis de mesa, atletismo, judô e esgrima.

Os questionários foram aplicados na Universidade $\mathrm{Fe}$ deral de São Paulo (Unifesp) e Universidade Federal de Pernambuco (UFPE), de maneira individual e em salas específicas. As variáveis avaliadas nos questionários foram: padrão e queixas relativas ao sono, cronotipo e adaptação ao fuso horário.

O padrão e queixas de sono foram analisados a partir da aplicação do Questionário de Queixas de Sono ${ }^{18}$, que visa avaliar os seguintes aspectos:

- Satisfação com o sono; satisfação com o local onde dorme; dificuldades ao dormir; consumo de drogas; prejuízo nos últimos dois anos por problemas de sono; sonolência visível a terceiros; possíveis desordens durante o sono (sonambulismo, bruxismo, apnéia, asma, taquicardia, dor de cabeça, refluxo gástrico, ronco, cãibras, e PLM).

Juntamente foi aplicado o diário de sono ${ }^{19}$ de sete dias demonstrando as características individuais do seu cotidiano.

A avaliação cronotipica individual dos atletas foi realizada segundo o questionário de Horne e Östberg ${ }^{9}$, que nos fornece a classificação em relação a matutinidade e vespertinidade seguindo o seguinte escore: 16 a 33 - vespertino; 34 a 44 - vespertino moderado; 45 a 65 - indiferente; 66 a 76 - matutino moderado; 77 a 86 - matutino.

Os atletas também passaram por um cronograma de ressincronização ao fuso horário de Sidney, pois haveria um avanço de fase de 13 horas. Assim, foi orientado durante a viagem o horário em que se deveria dormir e ficar acordado, tendo sido administrado a melatonina $(3 \mathrm{mg})$ a toda delegação, uma hora antes do horário que se deveria dormir, no intuito de sinalizar ao nosso organismo o novo horário para dormir e assim propiciar uma adequação a alteração e arrastamento do ritmo biológico de toda a delegação.

Após as avaliações os atletas receberam um relatório individual que continha os resultados da análise dos questionários de queixas de sono, matutinidade e vespertinidade e do diário de sono de sete dias.

\section{RESULTADOS}

Os resultados apresentados nas tabelas abaixo (1-7) demonstram as avaliações referentes às características gerais dos atletas paraolímpicos, a avaliação do padrão e queixas de sono, a avaliação do cronotipo e a proposta para a sincronização ao fuso horário de Sidney.

TABELA 1

Dados gerais dos atletas paraolímpicos avaliados

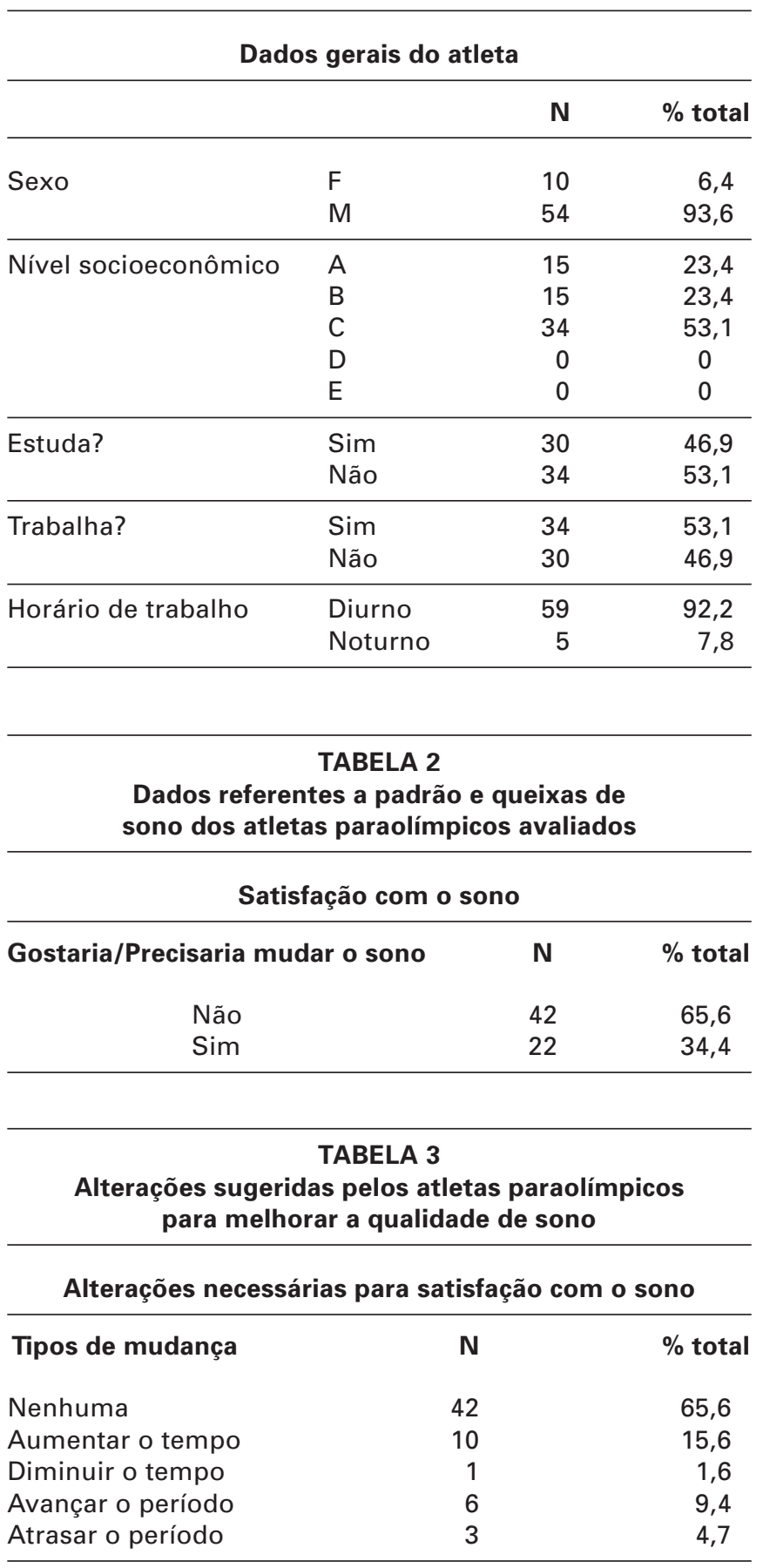


TABELA 4

Incidência de insônia da população estudada

\begin{tabular}{|c|c|c|c|c|c|c|c|c|}
\hline \multicolumn{9}{|c|}{ Dificuldades ao dormir } \\
\hline & \multicolumn{2}{|c|}{$\begin{array}{l}\text { Dificuldade de } \\
\text { iniciar o sono }\end{array}$} & \multicolumn{2}{|c|}{$\begin{array}{l}\text { Insônia de } \\
\text { fim de noite }\end{array}$} & \multicolumn{2}{|c|}{$\begin{array}{l}\text { Despertares } \\
\text { noturnos }\end{array}$} & \multicolumn{2}{|c|}{$\begin{array}{c}\text { Sonolência } \\
\text { excessiva diurna }\end{array}$} \\
\hline & $\mathbf{N}$ & $\%$ total & $\mathbf{N}$ & $\%$ total & $\mathbf{N}$ & $\%$ total & $\mathbf{N}$ & $\%$ total \\
\hline Nenhum & 39 & 60,9 & 46 & 71,9 & 51 & 79,7 & 58 & 96,6 \\
\hline Raramente & 7 & 10,9 & 8 & 12,5 & 2 & 3,1 & 4 & 6,3 \\
\hline 2 a 3 vezes/mês & 1 & 1,6 & 0 & 0 & 1 & 1,6 & 0 & 0 \\
\hline 1 a 2 vezes/semana & 4 & 6,3 & 4 & 6,3 & 2 & 3,1 & 0 & 0 \\
\hline 3 a 6 vezes/semana & 8 & 12,5 & 1 & 1,6 & 3 & 4,7 & 1 & 1,6 \\
\hline Sempre & 5 & 7,8 & 4 & 6,3 & 5 & 7,8 & 1 & 1,6 \\
\hline
\end{tabular}

TABELA 5

Dados referentes à freqüência dos distúrbios do sono nos atletas paraolímpicos avaliados

\begin{tabular}{|c|c|c|c|c|c|c|c|c|c|c|c|c|}
\hline \multicolumn{13}{|c|}{ Freqüência dos distúrbios do sono nos atletas paraolímpicos } \\
\hline \multirow[t]{2}{*}{ Distúrbios } & \multicolumn{2}{|c|}{ Nunca } & \multicolumn{2}{|c|}{$\begin{array}{l}\text { Rara- } \\
\text { mente }\end{array}$} & \multicolumn{2}{|c|}{$\begin{array}{c}2 \text { a } 3 \\
\text { vezes/ } \\
\text { mês }\end{array}$} & \multicolumn{2}{|c|}{$\begin{array}{c}1 \text { a } 2 \\
\text { vezes/ } \\
\text { semana }\end{array}$} & \multicolumn{2}{|c|}{$\begin{array}{c}3 \text { a } 6 \\
\text { vezes/ } \\
\text { semana }\end{array}$} & \multicolumn{2}{|c|}{ Sempre } \\
\hline & $\mathbf{N}$ & $\%$ & $\mathbf{N}$ & $\%$ & $\mathbf{N}$ & $\%$ & $\mathbf{N}$ & $\%$ & $\mathbf{N}$ & $\%$ & $\mathbf{N}$ & $\%$ \\
\hline Sonambulismo & 62 & 96,9 & 2 & 3,1 & 0 & 0 & 0 & 0 & 0 & 0 & 0 & 0 \\
\hline Bruxismo & 57 & 89,1 & 6 & 9,4 & 0 & 0 & 1 & 1,6 & 0 & 0 & 0 & 0 \\
\hline Apnéia & 55 & 85,9 & 9 & 14,1 & 0 & 0 & 0 & 0 & 0 & 0 & 0 & 0 \\
\hline Epilepsia & 63 & 98,4 & 0 & 0 & 1 & 1,6 & 0 & 0 & 0 & 0 & 0 & 0 \\
\hline Asma & 62 & 96,9 & 1 & 1,6 & 1 & 1,6 & 0 & 0 & 0 & 0 & 0 & 0 \\
\hline Pânico noturno & 57 & 89,1 & 6 & 9,4 & 0 & 0 & 1 & 1,6 & 0 & 0 & 0 & 0 \\
\hline Taquicardia & 63 & 98,4 & 1 & 1,6 & 0 & 0 & 0 & 0 & 0 & 0 & 0 & 0 \\
\hline Refluxo gástrico & 54 & 84,4 & 10 & 15,6 & 0 & 0 & 0 & 0 & 0 & 0 & 0 & 0 \\
\hline Dor de cabeça & 54 & 84,4 & 9 & 14,1 & 0 & 0 & 1 & 1,6 & 0 & 0 & 0 & 0 \\
\hline Ansiedade pós-pesadelo & 37 & 57,8 & 25 & 39,1 & 1 & 1,6 & 0 & 0 & 1 & 1,6 & 0 & 0 \\
\hline Ronco & 44 & 68,8 & 4 & 6,3 & 1 & 1,6 & 7 & 10,9 & 2 & 3,1 & 6 & 9,4 \\
\hline Cãibras & 51 & 79,7 & 13 & 20,3 & 0 & 0 & 0 & 0 & 0 & 0 & 0 & 0 \\
\hline Sonilóquio & 45 & 70,3 & 17 & 26,6 & 0 & 0 & 0 & 0 & 2 & 3,1 & 0 & 0 \\
\hline PLM & 54 & 84,4 & 6 & 9,4 & 0 & 0 & 2 & 3,1 & 1 & 1,6 & 1 & 1,6 \\
\hline
\end{tabular}

\section{DISCUSSÃO}

Observa-se que mesmo com um alto índice relativo à insatisfação com o seu próprio sono $(34,4 \%)$, quando questionado sobre a necessidade de alterar ou mudar o seu sono, os atletas não apresentaram um percentual elevado de queixas relativas ao sono, quando questionados especificamente sobre esses distúrbios. No entanto, observa-se que não há uma boa compreensão, por parte dos avaliados, sobre a necessidade de se dormir bem, muito menos sobre a importância de se ter uma boa higiene do sono.

Outro aspecto importante é a necessidade de manter esse modelo de avaliação, uma vez que todo o processo restau- rador, seja físico e ou cognitivo do ser humano é deflagrado e executado com maior ênfase durante o sono, já que durante o sono REM/Paradoxal temos uma restauração cognitiva, melhorando os aspectos relacionados a memória, aprendizado entre outros, e durante o sono Delta/Profundo e ou estágios 3 e 4 do sono, enfatiza-se a restauração tecidual periférica prevalecendo uma restauração do sistema músculo-esquelético, entre outras.

O desenvolvimento de atividades teóricas com os atletas, na tentativa de sensibilizar os mesmos para que todos viessem a ter uma melhora na qualidade e eficiência do sono foi de suma importância, pois durante toda a compe- 
TABELA 6

Dados referentes à sugestão à sincronização ao fuso horário de Sidney apresentada ao CPB (que deveria ter ocorrido na cidade de Curitiba (PR) no período que antecedesse a viagem a Sidney)

\begin{tabular}{|c|c|c|c|c|c|c|c|}
\hline \multicolumn{8}{|c|}{ Agenda dos atletas no período pré-viagem para Sydney } \\
\hline \multirow{2}{*}{$\begin{array}{l}\text { Atividades } \\
\text { pré-viagem }\end{array}$} & \multicolumn{7}{|c|}{ Horário Brasil } \\
\hline & $2 / 10 / 00$ & $3 / 10 / 00$ & 4 e $5 / 10 / 00$ & $6 / 10 / 00$ & $7 / 10 / 00$ & $\begin{array}{l}\text { 8/10/00 } \\
\text { horário } \\
\text { verão }\end{array}$ & $\begin{array}{l}\text { 9/10/00 } \\
\text { horário } \\
\text { verão }\end{array}$ \\
\hline Acordar & Livre & $7 \mathrm{~h}$ & $9 \mathrm{~h}$ & $9 \mathrm{~h}$ & $11 \mathrm{~h}$ & 12 & 12 \\
\hline Desjejum & $8 \mathrm{~h}$ & $8 \mathrm{~h}$ & $10 \mathrm{~h}$ & $10 \mathrm{~h}$ & $12 \mathrm{~h}$ & $13 \mathrm{~h}$ & $13 \mathrm{~h}$ \\
\hline Treinamento/livre & $8 \mathrm{~h}$ às $12 \mathrm{~h}$ & $8 \mathrm{~h}$ às $12 \mathrm{~h}$ & $10 \mathrm{~h}$ às $14 \mathrm{~h}$ & $10 \mathrm{~h}$ às $14 \mathrm{~h}$ & $12 \mathrm{~h}$ às $16 \mathrm{~h}$ & $13 \mathrm{~h}$ às $17 \mathrm{~h}$ & $13 \mathrm{~h}$ às $17 \mathrm{~h}$ \\
\hline Almoço & $12 \mathrm{~h}$ & $12 \mathrm{~h}$ & $14 \mathrm{~h}$ & $14 \mathrm{~h}$ & $16 \mathrm{~h}$ & $17 \mathrm{~h}$ & $17 \mathrm{~h}$ \\
\hline Livre/treinamento & $12 \mathrm{~h}$ às $19 \mathrm{~h}$ & $12 \mathrm{~h}$ às $19 \mathrm{~h}$ & $14 \mathrm{~h}$ às $21 \mathrm{~h}$ & $14 \mathrm{~h}$ às $21 \mathrm{~h}$ & $16 \mathrm{~h}$ às $23 \mathrm{~h}$ & $17 \mathrm{~h}$ à $0 \mathrm{~h}$ & $17 \mathrm{~h}$ à $0 \mathrm{~h}$ \\
\hline Lanche & $15 \mathrm{~h}$ & $15 \mathrm{~h}$ & $17 \mathrm{~h}$ & $17: 30 \mathrm{~h}$ & $19 \mathrm{~h}$ & $20 \mathrm{~h}$ & $20 \mathrm{~h}$ \\
\hline Jantar & $19 \mathrm{~h}$ & $19 \mathrm{~h}$ & $21 \mathrm{~h}$ & $22 \mathrm{~h}$ & $23 \mathrm{~h}$ & $\mathrm{Oh}$ & Oh \\
\hline Livre/acordado & $19: 30 h$ às $21: 30 h$ & $19: 30 h$ às $21: 30 h$ & $21: 30 h$ às $23 h$ & $21: 30 h$ às $0: 30 h$ & $23 h$ à $1 h^{*}$ & $0 h$ às $2 \mathrm{~h}$ & $0 h$ às $3 h$ \\
\hline Lanche & $21: 30 h$ & $22 \mathrm{~h}$ & $23 \mathrm{~h}$ & $0: 30 h$ & $1 h^{*}$ & $2 \mathrm{~h}$ & $3 \mathrm{~h}$ \\
\hline Livre/acordado & $21: 30$ às $22 \mathrm{~h}$ & $22 \mathrm{~h}$ à $0 \mathrm{~h}$ & $23 \mathrm{~h}$ à $0 \mathrm{~h}$ & $0: 30 h$ às $2 \mathrm{~h}$ & $1 \mathrm{~h}$ às $2 h^{*}$ & $2 h$ às $3 h$ & $3 h$ às $4 h$ \\
\hline Dormir & $22 \mathrm{~h}$ & Oh & $\mathrm{Oh}$ & $2 \mathrm{~h}$ & $3 h^{*}$ & $3 \mathrm{~h}$ & $4 \mathrm{~h}$ \\
\hline
\end{tabular}

TABELA 7

Avaliação do cronotipo dos atletas paraolímpicos

\section{Classificação do cronotipo}

Vespertino típico

\begin{tabular}{rc}
0 & $0 \%$ \\
4 & $6,22 \%$ \\
47 & $73,43 \%$ \\
13 & $20,31 \%$ \\
0 & $0 \%$ \\
\hline
\end{tabular}

Vespertino moderado

Indiferente

Matutino moderado

Matutino típico

tição e translado entre o Brasil e Sidney, os atletas tiveram uma excelente participação e colaboração na adaptação ao fuso horário.

O processo de sincronização ao fuso horário foi realizado de forma abrupta, na tentativa de romper com o ciclo claro-escuro que estava relacionado ao horário brasileiro e imediatamente adaptar ao ciclo, no fuso horário de Sidney, uma vez que não houve oportunidade de "arrastar" o ciclo claro-escuro da forma que se havia planejado (anteriormente descrito - tabela 6). Assim, foi orientado durante a viagem o horário em que se deveria dormir e ficar acordado, tendo sido administrado a melatonina $(3 \mathrm{mg})$ a toda dele- gação, uma hora antes do horário que se deveria dormir e também, durante os sete primeiros dias após a chegada em Sidney, com a exposição a luminosidade (luz solar), durante o maior período possível, principalmente de 8:00h às $12: 00 \mathrm{~h}$ e $14: 00 \mathrm{~h}$ às $18: 00 \mathrm{~h}$, no intuito de sinalizar ao nosso organismo ao novo horário para iniciar o sono e assim propiciar uma adequação a alteração e arrastamento do ritmo biológico de todos os atletas e membros da delegação. Para os atletas e membros da delegação que não respondiam bem a melatonina ( $3 \mathrm{mg}$ ) foi utilizado um indutor do sono, não benzodiazepnico, uma droga de última geração da categoria "Imidas Zolpiridinas" (Zolpidem), que possui uma meia vida curta e que até o presente momento os estudos não demonstram uma alteração na arquitetura do sono, mantendo o percentual de estágios 3, 4 e REM/Paradoxal e tendo um baixo nível, ou quase nenhum, de dependência para os usuários. Todas essas recomendações somente foram possíveis após uma minuciosa avaliação do grupo e uma grande análise de toda a equipe médica do $\mathrm{CPB}$, da Equipe de Avaliação e do Instituto do Sono da Unifesp.

Em todo o processo de sincronização ou ressincronização ao fuso horário, estudos demonstram que o adianta- 
mento do relógio biológico é mais eficiente e menos traumático, pois o nosso organismo se prepara de forma mais rápida em tal procedimento. Já o processo inverso é sempre mais traumático, ou seja, "voltar no tempo", pois o mecanismo de adaptação biológica está mais preparado para um ciclo de 28 horas, sendo assim mais traumático toda a viagem contrária ao relógio biológico.

Outro processo importante de se observar é que um dos grandes marcadores biológicos que temos é a temperatura corporal e é exatamente essa adaptação (da temperatura ao novo ciclo claro-escuro) que faz com que o nosso organismo tenha todo o efeito do Jet Lag. Em geral, somente 25\% das pessoas não apresentam problemas com essa adaptação, no entanto, para os outros $75 \%$ da população esse efeito é devastador no organismo, ou seja, o indivíduo que cruza fusos de forma rápida fica totalmente alerta durante o ciclo escuro e com muita sonolência no período claro. Esse processo leva um grande prejuízo social, físico e cognitivo durante essa fase de sincronização.

Pensando nesse aspecto é que a comissão de avaliação se preocupou com todo esse processo de adaptação ao fuso horário, pois a grande preocupação não era somente a adaptação e adequação do fuso horário para os dias da competição (jogos paraolímpicos), pois chegaríamos com tempo suficiente para uma adaptação sem grandes transtornos (em geral um dia para cada fuso alterado). Mas nossa atenção estava voltada para o período de treinamento, uma vez que caso essa adaptação não ocorresse de forma adequada todo o período de treinamento pré-competição poderia estar comprometido, e correríamos o risco dos atletas diminuírem e ou reduzirem, mais do que o necessário a relação entre a intensidade e o volume do treinamento e assim chegassem na data da competição com um percentual de carga e/ou estímulo de trabalho reduzido, podendo prejudicar os resultados.

Outro aspecto importante foi o de tentar aumentar as fases 3 e 4 do sono e conseqüentemente a liberação de $\mathrm{GH}$ (hormônio do crescimento) durante estas fases através da utilização de melatonina, tentando assim auxiliar no processo endógeno de recuperação obtido nesta fase do sono.

Contudo, podemos observar que todo o trabalho teve uma boa aceitação e deve ter contribuído e refletido nos resultados finais dos jogos (seis medalhas de ouro, 10 medalhas de prata e seis medalhas de bronze, foi a melhor participação brasileira nos jogos paraolímpicos). No entanto é de fundamental importância a continuidade de toda essa avaliação, inclusive para competições que não se necessite de uma grande alteração e adaptação ao fuso horário, pois o intuito de melhorar não só a qualidade, padrão e eficiência do sono mas também de garantir um bom processo de recuperação e melhoria das capacidades físicas e cognitivas restauradas durante o sono que são estimuladas e utilizadas durante o treinamento físico, técnico e tático.

\section{AGRADECIMENTOS}

- Comitê Paraolímpico Brasileiro (CPB)

- Secretaria Nacional de Esportes (MET)

- Cepid/Sono - Fapesp/Unifesp

- Cenesp/Unifesp (Rede Cenesp)

- Banco do Brasil

- Associação de Fundo e Incentivo à Psicofarmacologia (Afip)

- Universidade Federal de São Paulo

- Universidade de Pernambuco

- Centro de Estudos em Psicobiologia e Exercício (Cepe) da Unifesp

\section{REFERÊNCIAS}

1. Rechtschaffen A, Kales A. Manual of standardized terminology, techniques, and scoring system for sleep stages of human subjects. Brain Information Service/Brain Research Institute, UCLA, Los Angeles, 1968.

2. American Sleep Disorders Association. The international classification of sleepdisorders (diagnostic and coding manual). Kansas, DCSC, 1991.

3. Halberg F. Chronobiology. Annual Review of Physiology 1969;31:675-725.

4. Aschoff J. Circadian rhythms in man. Science 1965;148:1427-32.

5. Marques MD. Adaptação temporal. In: Marques, N.; Menna-Barreto, L., orgs. Cronobiologia: Princípios e Aplicações. São Paulo, EDUSP, 1997;45-84.

6. Horne JA, Ostberg O. A self-assesment questionnaire to determine morningness/eveningness in human circadian rhythms. Int J Chronobiol 1976; 4:97-110

7. De Mello MT, Lauro FAA, Silva AC, Tufik S. Sleep study after acute physical activity in spinal cord injury. Sleep Res 1995;24A:391 (Abstract).

8. De Mello MT, Lauro FA, Silva AC, Tufik S. Incidence of periodic leg movements and of the restless legs syndrome during sleep following acute physical activity in spinal cord injury subjects. Spinal Cord 1996;34:294-6.

9. Lee MS, Choi YC, Lee SH, Lee SB. Sleep-related periodic leg movemet associated with spinal cord lesions. Movement Desorders 1996;11:719-22.

10. Yokota T, Hirose K, Tanabe H, Tsukagoshi H. Sleep-related periodic leg movements (nocturnal myoclonus) due to spinal cord lesion. J Neurol Sci 1991;104:13-8.

11. Lancioni GE, O'reilly MF, Basili G. Review of strategies for treating sleep problems in persons with severe or profound mental retardation or multiple handicaps. Am J Ment Retard 1999;104:170-86.

12. Espie CA, Paul A, McFie J, Amos P, Hamilton D, Mc Coll JH, Tarassenko L, Pardey J. Sleep studies of adults with severe or profound mental retardation and epilepsy. Am J Mental Retard 1998;103:47-59.

13. Shapiro CM, Griesel RD, Bartel PR, Jooste PL. Sleep patterns after graded exercise. J Appl Physiol 1975;39:187-90.

14. Heinzelmann F, Bagley R. Response to physical activity programs and their effects on health behavior. Public Health Report 1970;85:905-11.

15. Vuori I, Urponen H, Hasan J, Partinen M. Epidemiology of exercise effects on sleep. Acta Physiol Scand 1988;574:3-7.

16. Trinder J, Montgomery I, Paxton SJ. The effect of exercise on sleep: the negative view. Acta Physiol Scand 1988;574:14-20

17. Youngstedt SD, O'Connor PJ, Dishman RK. The effects of acute exercise on sleep: a quantitative synthesis. Sleep 1997;20:203-14.

18. Del Giglio SB. Estudo da Ocorrência de queixas de insônia, de sonolência excessiva diurna e das relativas ás parassonias na população adulta da cidade de São Paulo. 1988. Tese (Doutorado) - Escola Paulista de Medicina, São Paulo.

19. Andrade MM, Benedito-Silva AA, Domenice S, Arnhold IJ, Menna-Barreto L. Sleep characteristics of adolescents: a longitudinal study. J Adolesc Health 1993;14:401-6. 